\title{
Health Maintenance and Vaccination of Patients With Inflammatory Bowel Disease: Practice and Perception of Responsibility of Gastroenterologists vs Primary Care Providers
}

\author{
Muhammad B. Hammami, MD, ${ }^{1,2}$ Pratik Pandit, MD, ${ }^{2}$ Rebecca T. Salamo, MD, ${ }^{3}$ Florence-Damilola Odufalu, MD, ${ }^{1,2}$ \\ Katie Schroeder, MD' \\ ${ }^{1}$ Division of Gastroenterology and Hepatology, Saint Louis University School of Medicine, St. Louis, MO ${ }^{2}$ Department of Internal Medicine, \\ Saint Louis University School of Medicine, St. Louis, MO ${ }^{3}$ Saint Louis University School of Medicine, St. Louis, MO
}

Background: Although it is well established that patients with inflammatory bowel disease (IBD) are at increased risk of complicating diseases and vaccination-preventable infections, whether gastroenterologists (GIs) or primary care providers (PCPs) assume responsibility for these patients' health maintenance is not clear.

Methods: We anonymously surveyed a convenience sample of 94 PCPs and 61 Gls at Saint Louis University School of Medicine in St. Louis, MO, about their practice and perception of the health maintenance and vaccination of patients with IBD.

Results: Response rates were $82 \%$ and $93 \%$ for Gls and PCPs, respectively. Gls were as likely as PCPs to screen for smoking ( $88 \%$ vs $89 \%$ ) and were significantly less likely to screen for depression/anxiety ( $24 \%$ vs $54 \%$ ) or to provide pertussis ( $14 \%$ vs $44 \%$ ) or diphtheria ( $20 \%$ vs $48 \%$ ) vaccines. Gls were significantly more likely than PCPs to assess for colonoscopy need ( $94 \%$ vs $80 \%)$; to screen for nonmelanoma skin cancer ( $62 \%$ vs $14 \%)$, melanoma (56\% vs $7 \%$ ), osteoporosis ( $72 \%$ vs $51 \%)$, or tuberculosis (94\% vs 44\%); to prescribe calcium/vitamin D (74\% vs 53\%); to perform nutritional assessment (78\% vs 33\%); or to provide hepatitis A $(60 \%$ vs $39 \%)$ or hepatitis B ( $86 \%$ vs $56 \%)$ vaccines. Gls were as likely as PCPs $(64 \%$ vs $75 \%)$ to perceive that PCPs should order vaccinations and significantly more likely to perceive that Gls should track vaccinations (58\% vs $16 \%$ ) and other health maintenance issues ( $90 \%$ vs $49 \%)$. We found positive associations between performing the various health maintenance and vaccination tasks and the perception of responsibility.

Conclusion: Several health maintenance aspects are inadequately addressed by Gls and PCPs, in part because of conflicting perceptions of responsibility. Clear guidelines and better GI/PCP communication are required to ensure effective health maintenance for patients with IBD.

Keywords: Delivery of health care, inflammatory bowel diseases, risk, vaccination

Address correspondence to Katie Schroeder, MD, Division of Gastroenterology and Hepatology, Saint Louis University School of Medicine, 1402 S. Grand Blvd., St Louis, MO 63104. Tel: (314) 577-8764. Email: katie.schroeder@health.slu.edu

\section{INTRODUCTION}

Inflammatory bowel disease (IBD) is a spectrum of diseases that includes Crohn disease and ulcerative colitis. ${ }^{1}$ Although IBD primarily targets the gastrointestinal tract, it is associated with several extraintestinal manifestations that require timely screening and management. ${ }^{2}$

Treatment goals for patients with IBD have transitioned from solely symptomatic remission to mucosal and histochemical remission with an increasing use of early and aggressive therapies, including biologic agents and immune modulators. ${ }^{3}$ Patients with IBD have a dysregulated immune system that puts them at increased risk of complicating illnesses, ${ }^{2}$ and it is exaggerated by the use of biologic agents and immune modulators. Kantsø et al showed an increased risk of pneumococcal pneumonia in patients with IBD independent of IBD-specific medications. ${ }^{4}$ In addition, patients with IBD are at increased risk for nutritional deficiencies, osteoporosis, melanoma, nonmelanoma skin cancers, cervical cancer, depression, and anxiety. ${ }^{2}$ Further, given their immunocompromised status, these patients require special care regarding vaccinations. ${ }^{2}$

Patients with IBD often receive all their medical care from their gastroenterologist (GI) or an IBD specialist. ${ }^{5}$ Although several guidelines for health maintenance of patients with 
IBD have been published, ${ }^{2,6}$ patients with IBD may not be receiving routine preventive care at the same rate as general medical patients, ${ }^{7}$ perhaps in part because of ambiguity about whether primary care providers (PCP) or Gls should assume responsibility for these patients' health maintenance issues.

The aim of this study was to explore the practice and views of PCPs and Gls regarding the health maintenance issues, including vaccinations, of patients with IBD.

\section{METHODS}

For this prospective study, we anonymously surveyed a convenience sample of 94 PCPs and 61 Gls who are affiliates or trainees at Saint Louis University School of Medicine in St. Louis, MO, using a paper or an electronic self-administered questionnaire. To maintain anonymity, the electronic responses were delinked from participants' identifying information, including email address, IP address, and date and time of participation. The paper questionnaires were distributed to participants in group sessions. Participants completed them on their own time, and to maintain anonymity, deposited the completed questionnaires in a drop box. The study was undertaken as a quality improvement project and was exempt from institutional review board approval.

The authors developed the study questionnaire based on the 2017 American College of Gastroenterology (ACG) Clinical Guideline: Preventive Care in Inflammatory Bowel Disease. $^{2}$ During the development phase, we wanted to ensure that the questionnaire items would be understood by respondents as intended and that we had covered all intended areas of information. We iteratively evaluated the questionnaire by means of focused probing following completion of the questionnaire. In total, 8 respondents were interviewed: 4 during face validity assessment and 4 during pilot testing of the final version (for acceptability, comprehensibility, and response stability after 2-3 days). We added 1 item and reworded 4 items during the face validity assessment phase but did not make any changes during pilot testing.

The final questionnaire included 31 questions (Figure). Seventeen questions were related to vaccination, and 14 were related to other health maintenance issues. Questions explored perceived responsibility, frequency of performing various health maintenance tasks, comfort with giving vaccinations, and the vaccines for which patients with IBD should routinely be assessed.

For data analysis, we combined the responses "always" and "most of the time." Data are reported as frequencies (percentages) and were compared using chi-square test. Statistical significance was defined as $P \leq 0.05$. Unadjusted 2 -tailed $P$ values and 95\% confidence intervals are reported.

\section{RESULTS}

The response rate was $82 \%$ and $93 \%$ for Gls and PCPs, respectively. Twenty-four percent of the 50 participant Gls were university faculty, $14 \%$ were Veterans Affairs (VA) faculty, $40 \%$ were recent Gl graduates, and $22 \%$ were Gl fellows in training. Eleven percent of the 87 participant PCPs were university faculty, $7 \%$ were VA faculty, and $82 \%$ were medical residents.

\section{Health Maintenance of Patients With Inflammatory Bowel Disease: Reported Practice}

As shown in Table 1, various health maintenance activities were reportedly performed always/most of the time by $24 \%$ (screen for depression/anxiety) to $98 \%$ (check routine laboratory investigations in patients on immune modulators) of Gls and by $5 \%$ (enroll in a skin protection/surveillance program) to $89 \%$ (screen for tobacco use/counsel to quit) of PCPs.

No significant difference was found between the 2 groups in reported screening for smoking/counseling to quit. PCPs were more likely to report always/most of the time assessing for depression/anxiety $(P=0.0007)$. However, Gls were more likely to report always/most of the time assessing for IBD-related health maintenance issues $(P<0.0001)$, enrolling patients in skin protection/surveillance programs $(P<0.0001)$, screening for melanoma $(P<0.0001)$, screening for nonmelanoma skin cancers when patients are on immune modulators $(P<0.0001)$, assessing the need for surveillance colonoscopy $(P=0.03)$, prescribing calcium/vitamin $\mathrm{D}$ to patients on oral corticosteroids $(P=0.02)$, screening for osteoporosis by bone mineral density measurement $(P=0.02)$, screening patients on anti-tumor necrosis factor therapy for tuberculosis $(P<0.0001)$, performing routine nutritional assessments $(P<0.0001)$, and checking routine laboratory investigations for patients taking immune modulators $(P<0.0001)$.

\section{Vaccinations of Patients With Inflammatory Bowel Disease: Reported Practice}

As shown in Table 2, various vaccination-related tasks were reportedly performed always/most of the time by $10 \%$ (warn immune-compromised patients against handling diapers of rotavirus-vaccinated infants for a period of 4 weeks) to $86 \%$ (assess for hepatitis-B vaccine) of Gls and by $6 \%$ (warn immune-compromised patients against handling diapers of rotavirus-vaccinated infants for a period of 4 weeks) to $91 \%$ (provide annual influenza vaccine) of PCPs. No significant differences were found between the 2 groups in assessing vaccination status or in vaccination treatment plans for influenza, varicella/zoster, meningococcal, human papilloma virus, measles-mumps-rubella, and exposure to rotavirus.

PCPs were more likely to report assessing for pertussis $(P=0.0003)$ and diphtheria $(P=0.001)$ vaccines, selecting inactivated influenza vaccine if the patient was on immunesuppressive therapy $(P=0.02)$, and providing immunesuppressed patients with a pneumococcal conjugated vaccine (PCV13 or Prevnar 13), followed by a pneumococcal polysaccharide vaccine (PPSV23 or Pneumovax) $\geq 8$ weeks later and a booster every 5 years $(P=0.004)$. However, Gls were more likely to report assessing for hepatitis $A$ $(P=0.02)$ and hepatitis $\mathrm{B}(P=0.0003)$ vaccines and providing age-appropriate vaccinations prior to initiation of immunesuppressive therapy $(P=0.03)$.

\section{Health Maintenance of Patients With Inflammatory Bowel Disease: Perceived Responsibility}

Large and significant differences were found in perceived responsibility for health maintenance issues of patients with IBD between the 2 groups (Table 3). Overall, questionnaire 
Please complete the following questionnaire regarding patients with inflammatory bowel disease (IBD), ie, patients with Crohn disease or ulcerative colitis.

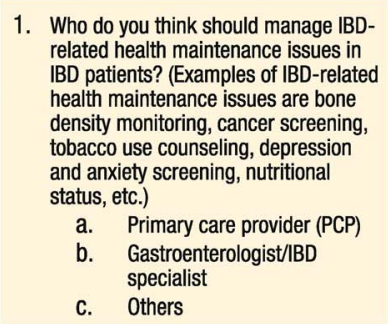

1. Who do you think should manage IBD related health maintenance issues in IBD patients? (Examples of IBD-related health maintenance issues are bone density monitoring, cancer screening, tobacco use counseling, depression and anxiety screening, nutritional status, etc.)

a. Primary care provider (PCP)

b. Gastroenterologist/IBD specialist

c. Others

2. Who do you think should keep track of IBD-related health maintenance issues? (Examples of IBD-related health maintenance issues are bone density monitoring, cancer screening tobacco use counseling, depression and anxiety screening, nutritional status, etc.)

a. Primary care provider (PCP)

b. Gastroenterologist/IBD specialist

c. Others

3. How often do you assess IBD-related health maintenance issues in your IBD patients? (Examples of IBD-related health maintenance issues are bone density monitoring, cancer screening, tobacco use counseling, depression and anxiety screening, nutritional status, etc.)
a. Always
b. Most of the time
c. Half of the time
d. Sometimes
e. Never

4. Who do you think should keep track of the vaccination status of IBD patients?

a. Primary care provider (PCP)

b. Gastroenterologist/IBD specialist

c. Others

5. Who do you think should be in charge of ordering/administering vaccines to IBD patients?

a. Primary care provider (PCP)

b. Gastroenterologist/IBD specialist

c. Others

6. How often do you assess the vaccination status of your IBD patients?

a. Always

b. Most of the time

c. Half of the time

d. Sometimes

e. Never

7. For which of the following vaccinations do you routinely assess your IBD patients? (Choose as many as are relevant.)

a. Hepatitis A

b. Hepatitis B

c. Meningococcal meningitis

d. Pertussis

e. HPV

f. Measles

g. Mumps

h. Rubella

i. Diphtheria
8. How often do you provide your IBD patients with age-appropriate vaccinations prior to initiation of immune suppression (or make sure it is provided)?

a. Always

b. Most of the time

c. Half of the time

d. Sometimes

e. Never

9. How comfortable are you in providing inactivated vaccines to your IBD patients?

a. Comfortable

b. Not comfortable

10. How comfortable are you in providing live vaccines (ie, measles, mumps, rubella, varicella, and herpes zoster) to your IBD patients?

a. Comfortable

b. Not comfortable

11. How often do you provide your IBD patients with an annual influenza vaccine (or make sure it is provided)?

a. Always

b. Most of the time

c. Half of the time

d. Sometimes

e. Never

12. How often do you make sure that the influenza vaccine provided to your IBD patients who are on immunosuppressive therapy is the inactivated and not the live vaccine?

a. Always

b. Most of the time

c. Half of the time

d. Sometimes

e. Never

13. How often do you make sure that the households of your IBD patients who are on immunosuppressive therapy are provided with the inactivated influenza vaccine and not the live one?

a. Always

b. Most of the time

c. Half of the time

d. Sometimes

e. Never

14. How often do you provide your IBD patients who are on immunosuppressive therapy with the PCV13 (Prevnar®) pneumonia vaccin followed by PPSV23 (Pneumovax@) $>8$ weeks later and a PPSV23 booster 5 years later (or make sure they are provided)?

a. Always

b. Most of the time

c. Half of the time

d. Sometimes

e. Never

15. How often do you provide the zoster (shingles) vaccine to your IBD patients who are over the age of 50 years?

a. Always

b. Most of the time

c. Half of the time

d. Sometimes

e. Never
16. How often do you provide your IBD patients who are over the age of 50 years and who are expected to start a biologic agent with the zoster (shingles) vaccine $>4$ weeks prior to starting the biologic agent?
a. Always
b. Most of the time
c. Half of the time
d. Sometimes
e. Never

17. How often do you check varicella virus (chickenpox) $\mathrm{lgG}$ and if negative consider vaccination for your IBD patients?
a. Always
b. Most of the time
c. Half of the time
d. Sometimes
e. Never

18. How often do you check varicella virus (chickenpox) $\operatorname{lgG}$ and if negative consider vaccination $>4$ weeks prior to starting a biologic agent for your IBD patients?
a. Always
b. Most of the time
c. Half of the time
d. Sometimes
e. Never

19. How often do you recommend to your immune-compromised IBD patients to avoid handling diapers of infants who have been vaccinated with rotavirus for 4 weeks after vaccination?

a. Always

b. Most of the time

c. Half of the time

d. Sometimes

e. Never

20. How often do you recommend to your immune-compromised IBD patients to avoid contact with persons who develop skin lesions after receiving varicella or zoster vaccines until the lesions clear?

a. Always

b. Most of the time

c. Half of the time

d. Sometimes

e. Never

21. How often do you assess for depression and anxiety in your IBD patients?

a. Always

b. Most of the time

c. Half of the time

d. Sometimes

e. Never

22. How often do you enroll your IBD patients in a skin protection/surveillance program?

a. Always

b. Most of the time

c. Half of the time

d. Sometimes

e. Never

23. How often do you screen your IBD patients for melanoma (perform a complete skin examination or refer to a dermatologist)?

a. Always

b. Most of the time

c. Half of the time

d. Sometimes

e. Never
24. How often do you screen your IBD patients who are on immune modulator therapy (6 MP or azathioprine) for nonmelanoma skin cancers (perform a complete skin examination or refer to a dermatologist)?

a. Always

b. Most of the time

c. Half of the time

d. Sometimes

e. Never

25. How often do you assess the need of your IBD patients for surveillance colonoscopy?

a. Always

b. Most of the time

c. Half of the time

d. Sometimes

e. Never

26. How often do you prescribe calcium/ vitamin $\mathrm{D}$ to your IBD patients who are on oral corticosteroids?

a. Always

b. Most of the time

c. Half of the time

d. Sometimes

e. Never

27. How often do you screen your IBD patients who have conventional risk factors for osteoporosis with a bone mineral density examination?
a. Always
b. Most of the time
c. Half of the time
d. Sometimes
e. Never

28. How often do you screen your IBD patients for tobacco use and/or counsel to quit?
a. Always
b. Most of the time
c. Half of the time
d. Sometimes
e. Never

29. How often do you screen your IBD patients who are on anti-TNF alpha therapy for tuberculosis?
a. Always
b. Most of the time
c. Half of the time
d. Sometimes
e. Never

30. How often do you perform a nutritional assessment (B12, folate, iron panel) for your IBD patients?

a. Always

b. Most of the time

c. Half of the time

d. Sometimes

e. Never

31. How often do you check routine labs (CBC, renal and hepatic function) to assess for side effects in your IBD patients who are on immune modulator therapy?

a. Always

b. Most of the time

c. Half of the time

d. Sometimes

e. Never

Figure. Study questionnaire (based on the 2017 American College of Gastroenterology Clinical Guideline: Preventive Care in Inflammatory Bowel Disease ${ }^{2}$ ). 


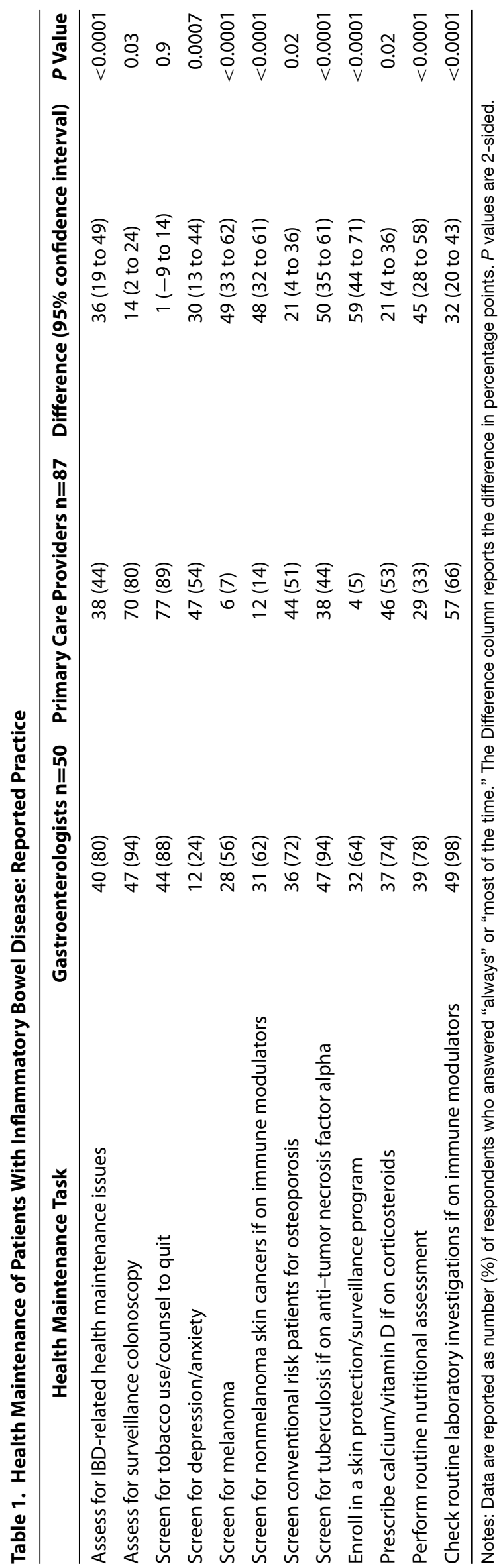

respondents perceived that Gls, not PCPs, should both manage $(P<0.0001)$ and track $(P<0.0001)$ IBD-related health maintenance issues.

\section{Vaccinations of Patients With Inflammatory Bowel Disease: Perceived Responsibility}

Although most Gls (64\%) and PCPs (75\%) had the perception that PCPs should order/administer vaccines with no significant difference between the 2 groups (Table 4), the groups were comfortable to similar degrees in providing inactivated (92\% of Gls and $84 \%$ of PCPs) and live attenuated (58\% of Gls and $56 \%$ of PCPs) vaccines.

However, as shown in Table 4, Gls were less likely to perceive that PCPs should keep track of vaccination status $(P<0.0001)$ and more likely to perceive that Gls should keep track of vaccination status $(P<0.0001)$.

\section{Association Between Reported Practice and Perception of Responsibility for Health Maintenance and Vaccinations of Patients With Inflammatory Bowel Disease}

To explore the association between practice and perceived responsibility, we divided the Gls and PCPs into subgroups according to their reported practice (task assessed always/most of the time vs half of the time/sometimes/never) and perception of responsibility (GI responsibility vs PCP responsibility) in regard to managing IBD-related health maintenance issues, tracking IBD-related health maintenance issues, ordering vaccines, and tracking vaccination status (ie, crossing question 3 with questions 1 and 2 and crossing question 6 with questions 4 and 5; refer to the Figure).

All associations were positive; ie, tasks were reportedly assessed always/most of the time more often when either group perceived them as their responsibility. Further, the results were significant in all 4 associations for PCPs and in 1 of the 4 associations for Gls (Table 5).

\section{DISCUSSION}

In our survey of the practice and views of university hospital-affiliated PCPs and Gls regarding health maintenance issues and vaccinations for patients with IBD, we found that $72 \%$ to $98 \%$ of Gls always/most of the time performed the recommended individual health maintenance tasks except for skin cancer surveillance and depression/anxiety screening that were reportedly performed by less than two-thirds and less than onefourth of respondents, respectively. PCP performance was similar to Gl performance in tobacco use, better in depression/anxiety screening, but worse otherwise. In contrast, except for hepatitis $A$, hepatitis $B$, influenza vaccines (providing annual influenza vaccine in general and selecting inactivated influenza vaccine for patients on immunosuppression), and providing age-appropriate vaccines prior to immunosuppression, $<50 \%$ of Gls (10\%-36\%) followed vaccination recommendations always or most of the time. Similarly, except for hepatitis $B$, influenza vaccines (providing annual influenza vaccines in general and selecting inactivated influenza vaccine for patients on immunosuppression), providing age-appropriate vaccines prior to immunosuppression, and providing appropriate pneumonia vaccine 


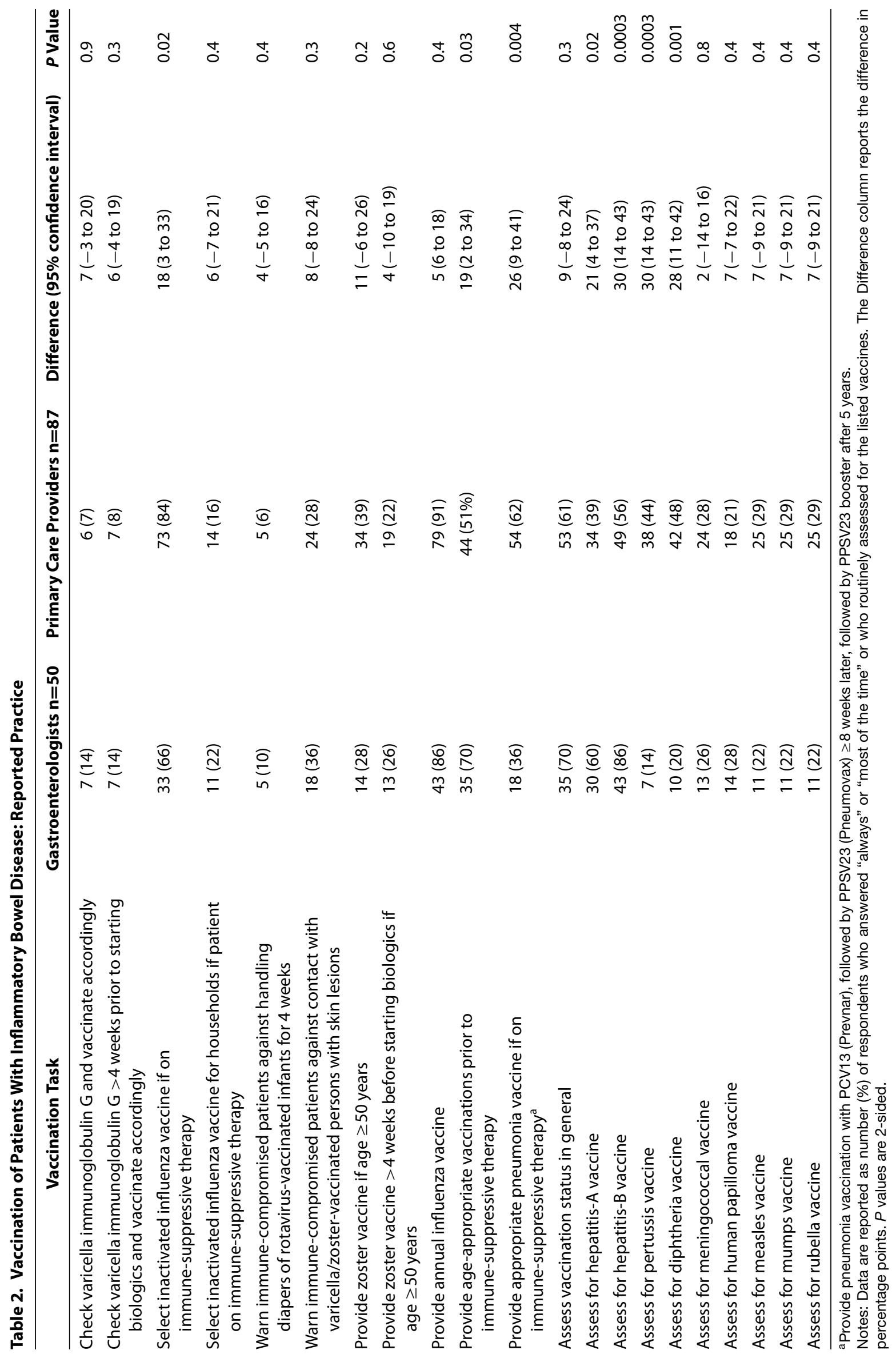



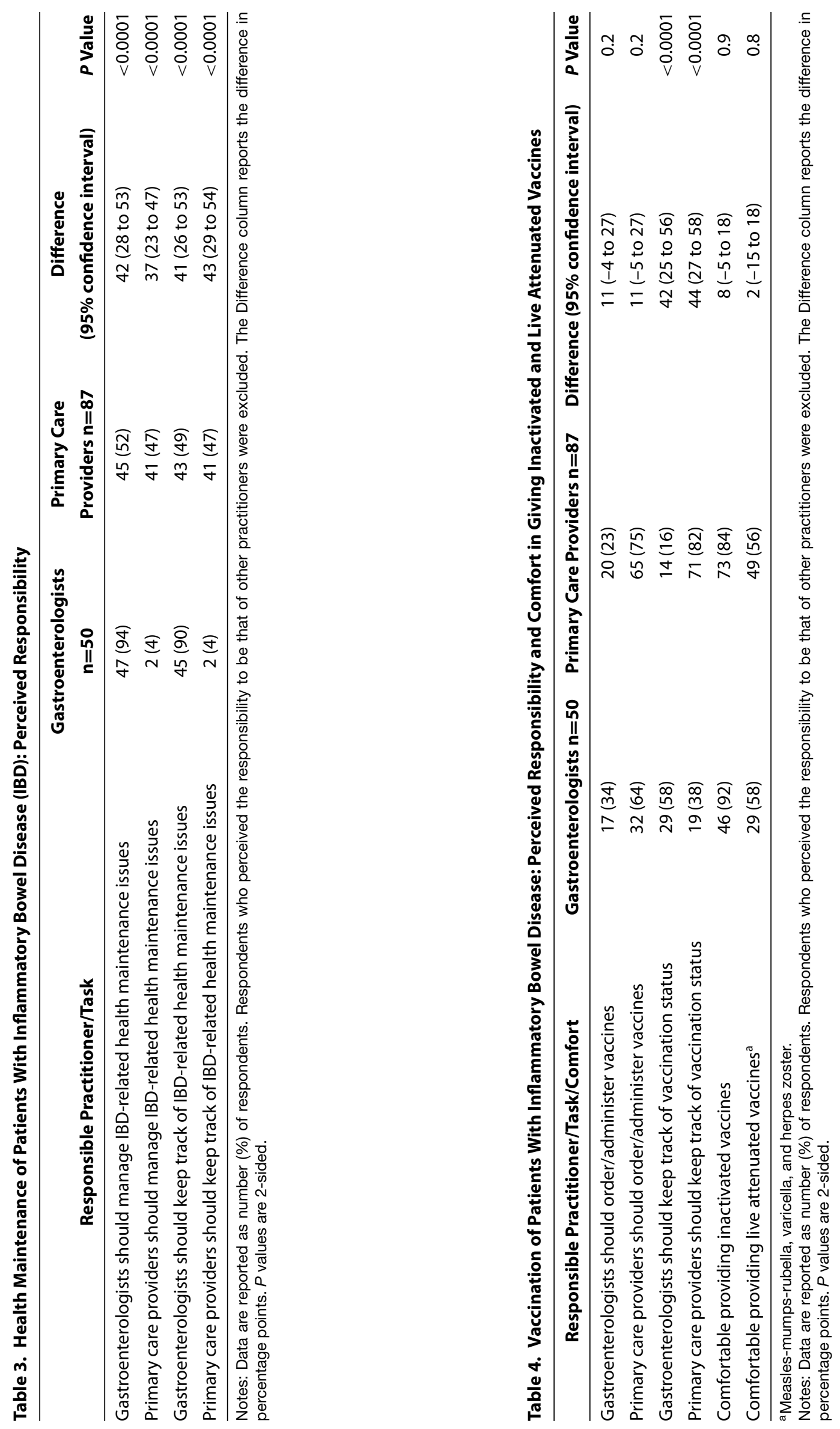
Table 5. Association Between Reported Practice and Perception of Responsibility for Health Maintenance and Vaccinations

\begin{tabular}{|c|c|c|c|}
\hline \multirow[b]{2}{*}{ Task/Respondent/Response } & \multicolumn{2}{|c|}{$\begin{array}{l}\text { Task Assessed Always/ } \\
\text { Most of the Time }\end{array}$} & \multirow[b]{2}{*}{$P$ Value } \\
\hline & Yes & No & \\
\hline \multicolumn{4}{|c|}{ Managing IBD-related health maintenance issues } \\
\hline GI respondents & & & 0.34 \\
\hline Gls are responsible & 38 & 9 & \\
\hline PCPs are responsible & 1 & 1 & \\
\hline PCP respondents & & & $<0.0001$ \\
\hline Gls are responsible & 5 & 40 & \\
\hline PCPs are responsible & 33 & 8 & \\
\hline \multicolumn{4}{|c|}{ Tracking IBD-related health maintenance issues } \\
\hline Gls respondents & & & 0.38 \\
\hline Gls are responsible & 36 & 9 & \\
\hline PCPs are responsible & 1 & 1 & \\
\hline $\mathrm{PCP}$ respondents & & & $<0.0001$ \\
\hline Gls are responsible & 2 & 41 & \\
\hline PCPs are responsible & 35 & 6 & \\
\hline \multicolumn{4}{|l|}{ Ordering vaccinations } \\
\hline GI respondents & & & 0.74 \\
\hline Gls are responsible & 13 & 4 & \\
\hline PCPs are responsible & 22 & 10 & \\
\hline $\mathrm{PCP}$ respondents & & & $<0.0001$ \\
\hline Gls are responsible & 3 & 17 & \\
\hline PCPs are responsible & 48 & 17 & \\
\hline \multicolumn{4}{|l|}{ Tracking vaccination status } \\
\hline GI respondents & & & 0.01 \\
\hline Gls are responsible & 24 & 5 & \\
\hline PCPs are responsible & 9 & 10 & \\
\hline PCP respondents & & & 0.006 \\
\hline Gls are responsible & 4 & 10 & \\
\hline PCPs are responsible & 49 & 22 & \\
\hline
\end{tabular}

Notes: Data are presented as number of respondents. Respondents who perceived the responsibility to be that of other practitioners were excluded. $P$ values are 2 -sided.

Gls, gastroenterologists; PCPs, primary care providers.

while on immunosuppression, $<50 \%$ of PCPs $(6 \%-48 \%)$ followed vaccination recommendations always or most of the time. Although most Gls and PCPs were reportedly comfortable providing inactivated vaccines, only $58 \%$ and $56 \%$, respectively, were comfortable providing live attenuated vaccines. Twenty-three percent and $52 \%$ of PCPs had the perception that Gls rather than PCPs are responsible for vaccination and IBD-related health maintenance, respectively. In contrast, $4 \%$, 38\%, and $64 \%$ of Gls perceived that PCPs are responsible for IBD-related health maintenance, keeping track of vaccination status, and administering vaccines, respectively. Finally, the reported adherence to recommendations was positively associated with the perception of responsibility in both groups.
Overall, the Gls' reported performance of health maintenance tasks in our study is acceptable (72\%-98\%), except for skin cancer surveillance (56\%-64\%) and depression/ anxiety screening (24\%). Patients with IBD are at increased risk for developing melanoma, ${ }^{8}$ and the risk is nearly double in the setting of anti-tumor necrosis factor therapy. ${ }^{9,10}$ IBD is not an independent risk factor for the development of nonmelanoma skin cancer; however, the risk of nonmelanoma skin cancer is increased with the use of thiopurines. ${ }^{11}$ The ACG recommends that all patients with IBD be counseled to decrease sun exposure by wearing protective clothing and using sunscreens with a sun protection factor of at least 30 and to undergo routine melanoma screening. In addition, patients with IBD who are taking immune modulators 
should be screened for nonmelanoma skin cancer, particularly patients who are $>50$ years of age. ${ }^{2}$

Bhandari et al found that patients with IBD were nearly twice as likely (49\% vs $23 \%$ ) to report depressive symptoms compared to patients without IBD, and IBD was a predictor of depressive symptoms. ${ }^{12}$ A systematic review found anxiety in $19 \%$ of patients with IBD compared to $10 \%$ of the background population and depression in $21 \%$ of patients with IBD compared to $13 \%$ in non-IBD controls. ${ }^{13}$ Depression rates as measured by the Hospital Anxiety and Depression Scale were similar in both active and inactive disease groups. ${ }^{13}$ Nigro et al found that the presence of depression or other psychiatric disorders in patients with IBD was significantly associated with medication noncompliance. ${ }^{14}$ As a conditional recommendation with low-level evidence, the ACG recommends screening patients with IBD for depression and anxiety. ${ }^{2}$

In contrast to their performance of health maintenance tasks, we found the Gls' reported performance of vaccination tasks to be poor overall. This finding is consistent with the results of a study showing that only $28 \%$ and $9 \%$ of 146 patients with IBD who were on current or previous immunosuppression received yearly influenza and pneumococcal vaccines, respectively. ${ }^{15}$ In another study, only $12 \%$ of 2,076 patients with IBD were vaccinated against hepatitis B virus, ${ }^{16}$ a rate much lower than the $86 \%$ reported assessment rate for hepatitis B vaccination in our study. Patients with IBD are at increased risk for preventable infections, so adherence to age-appropriate vaccination schedules is strongly recommended. ${ }^{2,15}$ In accordance with national guidelines, regardless of immunosuppression status, all adult patients with IBD should receive non-live vaccines, ${ }^{2,17-21}$ including trivalent inactivated influenza, pneumococcal (PCV13 and PPSV23), hepatitis $A$, hepatitis $B$, haemophilus influenzae $B$, human papilloma virus, tetanus, and pertussis vaccines. On the other hand, the live attenuated vaccines for patients with IBD have important restrictions. ${ }^{2,22}$ Per the Infectious Disease Society of America and current ACG guidelines, varicella and herpes zoster vaccines are recommended for patients with IBD who are on low level but not high level immunosuppression. 2,17 The ACG defines patients on low level immunosuppression as those with significant protein calorie malnutrition or those receiving (or who received in the previous 3 months) systemic corticosteroids equivalent to $20 \mathrm{mg}$ prednisone/day for $\geq 14$ days, methotrexate $\leq 0.4 \mathrm{mg} / \mathrm{kg} /$ week, azathioprine $\leq 3.0 \mathrm{mg} / \mathrm{kg} /$ day, or 6 -mercaptopurine $\leq 1.5 \mathrm{mg} / \mathrm{kg} /$ day. $^{2}$ On the other hand, the guidelines strongly suggest that varicella and herpes zoster vaccines be avoided in patients who have been on high-dose immunosuppressive therapy within the past 3 months or in patients who plan to start high-dose immunosuppressive therapy within the next 6 weeks. ${ }^{2,22}$ Although administering varicella or herpes zoster vaccines to household members of immunosuppressed patients is not contraindicated, if vaccine recipients develop a postvaccination rash, immunosuppressed patients should maintain contact precautions until rash resolution. ${ }^{2,17}$ The measles-mumps-rubella vaccine is contraindicated for patients who are receiving, who received in the previous 3 months, or who plan to receive within 6 weeks any immunosuppressive therapy. ${ }^{2}$

Potential reasons of noncompliance with guidelines include guideline unawareness, discomfort in performing unfamiliar tasks such as administering live vaccines to immune-suppressed patients, inadequate communication, and conflicting perceptions of responsibility among healthcare providers. One study demonstrated poor knowledge among 108 Gls about which vaccines to recommend to patients with IBD. ${ }^{23}$ Despite almost unanimously perceiving that they are responsible for managing IBD-related health maintenance issues, Gls were not compliant with skin cancer surveillance and depression/anxiety screening, suggesting inadequate guideline awareness.

In our study, PCPs outperformed Gls in tasks more related to primary care (such as screening for depression and anxiety and providing pneumonia, pertussis, and diphtheria vaccines), and Gls outperformed PCPs in tasks more specific to IBD and its management or to their subspecialty (such as screening for tuberculosis in patients on immune modulators and for osteoporosis in patients on oral corticosteroids and assessing for hepatitis $A$ and hepatitis $B$ vaccines). We also found a low comfort rate in providing live vaccines in association with a very low assessment rate for measlesmumps-rubella and varicella vaccination. Together, the data suggest that adherence to guidelines may be related to familiarity with the tasks. In this vein, Selby et al found that only $29 \%$ of family physicians were comfortable making vaccination recommendations for their patients with IBD. ${ }^{24}$

Alternatively, noncompliance with the guidelines may be attributable to conflicting perceptions of responsibility for the various health maintenance tasks among healthcare providers. This idea is supported by the association between adherence to recommendations and the perception of responsibility among PCPs and, to some extent, among Gls that was observed in the current study. For vaccines that are given in series, Gls may think that traveling to their specialist's office could be cumbersome for patients and because patients visit their PCPs more frequently, the PCP's office may be more suitable for managing such vaccinations. On the other hand, PCPs may have concerns about the complexity of managing patients with IBD, especially when disease activity is not under control or new medications are being started or titrated, and therefore may think the Gl's office may be more suitable for vaccination management.

Several measures have been shown to improve vaccination rates in patients with IBD, including the availability of vaccines in the Gl's office, education of healthcare professionals, ${ }^{25-27}$ and using checklists. ${ }^{28}$

\section{Limitations}

Limitations of this study include the relatively small sample size and sampling being restricted to affiliates of one university hospital. All 137 respondents were involved in training trainees from Saint Louis University School of Medicine or practiced/completed their training at Saint Louis University School of Medicine in either internal medicine or gastroenterology. In addition, many PCP respondents were current trainees. Thus, our results may not apply to other settings, as a lack of knowledge may have contributed to the results this setting. Also, we examined questionnaires rather than actual practice, and survey responses have an inherent, self-serving bias. However, such bias would be expected to strengthen rather than weaken the main conclusions of the study. 


\section{CONCLUSION}

We conclude that several health maintenance aspects for patients with IBD are inadequately addressed by Gls and PCPs, perhaps attributable in part to conflicting perceptions of responsibility. Guidelines that not only recommend tasks to be performed but also which party should perform the tasks may be required. In addition, better dissemination of guidelines and better GI/PCP communication and coordination, such as sharing medical documentation and using electronic alerts, are required for effective health maintenance in patients with IBD.

\section{ACKNOWLEDGMENTS}

The authors have no financial or proprietary interest in the subject matter of this article. Portions of the manuscript have been presented as abstracts at the following meetings: American College of Gastroenterology, Orlando, FL, October 2017; Advances in Inflammatory Bowel Disease, Orlando, FL, November 2017; and Crohn's and Colitis Congress, Las Vegas, NV, January 2018.

\section{REFERENCES}

1. Talley NJ, Abreu MT, Achkar JP, et al. An evidence-based systematic review on medical therapies for inflammatory bowel disease. Am J Gastroenterol. 2011 Apr;106 Suppl 1:S2-25; quiz S26. doi: 10.1038/ajg.2011.58.

2. Farraye FA, Melmed GY, Lichtenstein GR, Kane SV. ACG clinical guideline: preventive care in inflammatory bowel disease. $A m$ Gastroenterol. 2017 Feb;112(2):241-258. doi: 10.1038/ajg.2016.537.

3. Levesque BG, Sandborn WJ, Ruel J, Feagan BG, Sands BE, Colombel JF. Converging goals of treatment of inflammatory bowel disease from clinical trials and practice. Gastroenterology. 2015 Jan;148(1):37-51. doi: 10.1053/j.gastro.2014.08.003.

4. Kantsø B, Simonsen J, Hoffmann S, Valentiner-Branth $P$, Petersen AM, Jess T. Inflammatory bowel disease patients are at increased risk of invasive pneumococcal disease: a nationwide Danish cohort study 1977-2013. Am J Gastroenterol. 2015 Nov; 110(11):1582-1587. doi: 10.1038/ajg.2015.284.

5. Kane SV. Health maintenance assessment for patients with inflammatory bowel disease. Gastroenterol Hepatol (N Y). 2017 Aug;13(8):500-503.

6. Reich J, Wasan S, Farraye FA. Vaccinating patients with inflammatory bowel disease. Gastroenterol Hepatol (N Y). 2016 Sep;12(9):540-546.

7. Selby L, Kane S, Wilson J, et al. Receipt of preventive health services by IBD patients is significantly lower than by primary care patients. Inflamm Bowel Dis. 2008 Feb;14(2):253-258. doi: 10.1002/ibd.20266.

8. Singh S, Nagpal SJ, Murad MH, et al. Inflammatory bowel disease is associated with an increased risk of melanoma: a systematic review and meta-analysis. Clin Gastroenterol Hepatol. 2014 Feb;12(2):210-218. doi: 10.1016/j.cgh.2013.04.033.

9. Long MD, Martin CF, Pipkin CA, Herfarth HH, Sandler RS, Kappelman MD. Risk of melanoma and nonmelanoma skin cancer among patients with inflammatory bowel disease. Gastroenterology. 2012 Aug;143(2):390-399.e1. doi: 10.1053/j.gastro.2012.05.004.

10. McKenna MR, Stobaugh DJ, Deepak P. Melanoma and non-melanoma skin cancer in inflammatory bowel disease patients following tumor necrosis factor- $\alpha$ inhibitor monotherapy and in combination with thiopurines: analysis of the Food and Drug Administration adverse event reporting system. J Gastrointestin Liver Dis. 2014 Sep;23(3):267-271.

11. Ariyaratnam J, Subramanian V. Association between thiopurine use and nonmelanoma skin cancers in patients with inflammatory bowel disease: a meta-analysis. Am J Gastroenterol. 2014 Feb;109(2):163-169. doi: 10.1038/ajg.2013.451.

12. Bhandari S, Larson ME, Kumar N, Stein D. Association of inflammatory bowel disease (IBD) with depressive symptoms in the United States population and independent predictors of depressive symptoms in an IBD population: a NHANES study. Gut Liver. 2017 Jul 15;11(4):512-519. doi: $10.5009 / \mathrm{gnl} 16347$.

13. Mikocka-Walus A, Knowles SR, Keefer L, et al. Controversies revisited: a systematic review of the comorbidity of depression and anxiety with inflammatory bowel diseases. Inflamm Bowel Dis. 2016 Mar;22(3):752-762. doi: 10.1097/MIB.0000000000000620.

14. Nigro G, Angelini G, Grosso SB, Caula G, Sategna-Guidetti C. Psychiatric predictors of noncompliance in inflammatory bowel disease: psychiatry and compliance. J Clin Gastroenterol. 2001 Jan;32(1):66-68.

15. Melmed GY, Ippoliti AF, Papadakis KA, et al. Patients with inflammatory bowel disease are at risk for vaccine-preventable illnesses. Am J Gastroenterol. 2006 Aug;101(8):1834-1840.

16. Loras C, Saro C, Gonzalez-Huix F, et al. Prevalence and factors related to hepatitis $B$ and $C$ in inflammatory bowel disease patients in Spain: a nationwide, multicenter study. Am J Gastroenterol. 2009 Jan;104(1):57-63.

17. Rubin LG, Levin MJ, Ljungman $P$, et al. IDSA clinical practice guideline for vaccination of the immunocompromised host. Clin Infect Dis. 2014 Feb;58(3):e44-e100. doi: $10.1093 / \mathrm{cid} / \mathrm{cit} 684$.

18. Sands BE, Cuffari C, Katz J, et al. Guidelines for immunizations in patients with inflammatory bowel disease. Inflamm Bowel Dis. 2004 Sep;10(5):677-692.

19. Long MD, Gulati A, Wohl D, Herfarth H. Immunizations in pediatric and adult patients with inflammatory bowel disease: a practical case-based approach. Inflamm Bowel Dis. 2015 Aug;21 (8):1993-2003. doi: 10.1097/MIB.0000000000000395.

20. Kim DK, Bridges CB, Harriman KH; Centers for Disease Control and Prevention ( $C D C)$; Advisory Committee on Immunization Practices (ACIP); ACIP Adult Immunization Work Group. Advisory committee on immunization practices recommended immunization schedule for adults aged 19 years or older-United States, 2015. MMWR Morb Mortal Wkly Rep. 2015 Feb 6;64(4):91-92.

21. Kim DK, Bridges CB, Harriman KH; Advisory Committee on Immunization Practices. Advisory committee on immunization practices recommended immunization schedule for adults aged 19 years or older: United States, 2016. Ann Intern Med. 2016 Feb 2;164(3):184-194. doi: 10.7326/M15-3005.

22. Reich J, Wasan SK, Farraye FA. Vaccination and health maintenance issues to consider in patients with inflammatory bowel disease. Gastroenterol Hepatol (N Y). 2017 Dec;13(12):717-724.

23. Wasan SK, Coukos JA, Farraye FA. Vaccinating the inflammatory bowel disease patient: deficiencies in gastroenterologists knowledge. Inflamm Bowel Dis. 2011 Dec;17(12):2536-2540. doi: 10.1002/ibd.21667.

24. Selby L, Hoellein A, Wilson JF. Are primary care providers uncomfortable providing routine preventive care for 
inflammatory bowel disease patients? Dig Dis Sci. 2011 Mar;56(3):819-824. doi: 10.1007/s10620-010-1329-8.

25. Parker S, Chambers WL, Spangler C, et al. A quality improvement project significantly increased the vaccination rate for immunosuppressed patients with IBD. Inflamm Bowel Dis. 2013 Aug;19(9):1809-1814. doi: 10.1097/MIB.0b013e31828c8512.

26. Reich JS, Miller HL, Wasan SK, et al. Influenza and pneumococcal vaccination rates in patients with inflammatory bowel disease. Gastroenterol Hepatol (N Y). 2015 Jun;11(6):396-401.
27. Christensen KR, Steenholdt C, Buhl SS, et al. Systematic information to health-care professionals about vaccination guidelines improves adherence in patients with inflammatory bowel disease in anti-TNF alpha therapy. Am J Gastroenterol. 2015 Nov;1 10(11):1526-1532. doi: 10.1038/ajg.2015.162.

28. Health maintenance checklist for adult IBD patients. Crohn's \& Colitis Foundation. www.crohnscolitisfoundation.org/ science-and-professionals/programs-materials/ health-maintenance-checklist.pdf. Accessed September 1, 2018.

This article meets the Accreditation Council for Graduate Medical Education and the American Board of Medical Specialties Maintenance of Certification competencies for Patient Care, Medical Knowledge, Interpersonal and Communication Skills, and Practice-Based Learning and Improvement. 